\title{
A Novel 7 RNA-Based Signature for Prediction of Prognosis and Therapeutic Responses of Wild-Type BRAF Cutaneous Melanoma
}

\section{Ruizheng Sun}

Clinical Medicine Eight-year Program, Central South University

Yaozhong Liu

Department of Cardiology, the Second Xiangya Hospital, Central South University

Cheng Lei

Clinical Medicine Eight-year Program, Central South University

Zhenwei Tang

Department of Dermatology, Xiangya Hospital, Central South University

Lulixia Lu ( $\nabla$ lulixia@csu.edu.cn )

Department of Dermatology, Xiangya Hospital, Central South University

\section{Research Article}

Keywords: BRAF, melanoma, signature, TCGA, mTOR, prognosis

Posted Date: February 8th, 2022

DOI: https://doi.org/10.21203/rs.3.rs-1300994/v1

License: (c) (i) This work is licensed under a Creative Commons Attribution 4.0 International License. Read Full License 


\section{Abstract}

Background: The prognosis of wild-type BRAF cutaneous melanoma (WT Bf-CM) patients remains poor due to the lack of therapeutic options. However, few studies have investigated the factors contributing to the prognosis of WT Bf-CM patients.

Methods: In this paper, we proposed and validated a novel 7-RNA based signature to predict the prognosis of WT Bf-CM by analyzing the information from TCGA database.

Results: Dependence of this signature to other clinical factors were verified and a nomogram was also drawn to promote its application in clinical practice. Functional analysis suggested that the predictive function of this signature might attribute to the prediction of the up-regulation of RNA splicing, transcription, and cellular proliferation in the high-risk group, which have been demonstrated to be linked to malignancy of cancer. Moreover, therapy response analysis supported that this signature could predict the responses of $\mathrm{PI} 3 \mathrm{~K} / \mathrm{Akt} / \mathrm{mTOR}$ pathway based drugs in WT Bf-CM patients.

Conclusion: Collectively, this study will provide a preliminary bioinformatics evidence for the molecular mechanism and potential drug targets that could improving WT Bf-CM prognosis.

\section{Introduction}

Characterized by rapid progression and metastasis, cutaneous melanoma (CM) is considered as the most aggressive skin cancer, accounting for about 55500 death worldwide annually ${ }^{1}$. During the past decades, some breakthroughs have been made and significantly improve the survival of metastatic CM patients. For example, the clinical application of BRAF inhibitors and MEK inhibitors, which can extend the progression-free survival (PFS) of BRAF V600-mutate CM patients to 11-14.9 months ${ }^{1}$. However, CM patients carrying BRAF V600 mutations only makes up $45-50 \%$ of total melanoma patients ${ }^{2}$. For wildtype BRAF CM (WT Bf-CM) patients, that accounts for about $30 \%$ of total CM patients, the response rate to MEK inhibitor is merely $10 \%{ }^{3-5}$. Lack of therapeutic options resulted in the poor prognosis of WT Bf$\mathrm{CM}$. With a chemotherapy of dacarbazine, the median PFS of WT Bf-CM patients can be low as 1.5 months ${ }^{6}$.

Recently, with the help of next-generation sequencing (NGS) technology, the high heterogeneity of CM has been demonstrated, and it turns out that some features of this heterogeneity such as tumor mutational burden, are associate with the prognosis of $\mathrm{CM}^{7}$. Moreover, the high heterogeneity of $\mathrm{CM}$ indicates the existence of various biomarkers for prognosis. Although studies have revealed different biomarkers or signature for survival prediction, few of them have focused on the patients with wild-type BRAF $\mathrm{CM}^{8-10}$. Little is known about which factors can contribute to the poor prognosis of WT Bf-CM patients, apart from less therapy options, failing the need of precise management of WT Bf-CM patients.

To address this issue, this paper analyzed the whole transcription profiles of tumor tissues from 211 patients in the TCGA database, and proposed and validated a novel 7-RNA based signature to predict the 
prognosis of with wild-type BRAF CM. Moreover, functional analysis and therapy response analysis were also performed, providing a preliminary bioinformatics evidence for the molecular mechanism and potential drug targets that could improving WT Bf-CM prognosis.

\section{Materials And Methods}

\subsection{Data extraction}

The transcriptional profiles, mutation data and corresponding clinical information of $467 \mathrm{CM}$ patients were downloaded from TCGA-SKCM project with an R package "TCGAbiolinks" ${ }^{11}$. After excluding patients with BRAF somatic mutations and incomplete survival information, 211 WT Bf-CM patients' tumor samples were equally allocated into training group $(n=106)$ and testing group $(n=105)$. We also downloaded DNA methylation status of specific sites of the WT Bf-CM patients using R package "cgdsr". RNA-seq data were alignment to the human genome (Gencode.v22 annotation) and the expression of RNAs were measured by Reads Per Kilobase per Million mapped reads (RPKM). RNA expression profiles were identified based on the two following criteria: 1) transcripts were expressed in all WT Bf-CM samples; 2) average of RPKM > 0.1. Eventually, a total of 14202 RNAs in the profiles were enrolled.

\subsection{Statistical analysis}

In the training set, correlations between the expression of each RNA and overall survival (OS) of patients were calculated by univariate Cox regression and 251 RNAs with their P-values $<0.01$ were considered significant to patients' OS. Random forests were grown based on survival-related RNAs and variable hunting was implemented repeatedly using R package "randomForestSRC", which output top ranked variables by frequency of occurrence. 7 OS-relevant characteristics were selected as the best and fitted into stepped multivariate Cox regression analysis. Risk scores were weighted by the expression and coefficients of 7 RNAs. The median of risk scores in the training set served as a cut-off, respectively dividing WT Bf-CM patients into high risk groups and low risk groups both in the training set and the testing set. Kaplan-Meier (KM) analysis and visualization were performed to evaluate the survival differences between high risk groups and low risk groups by the "survival" R package. For WT Bf-CM patients with at least 5-year follow ups, receiver operating characteristic (ROC) curves were used to show the sensitivities and specialties of signatures' abilities to predict the 5-years OS using categorical variables. The area under ROC (AUC) scores and 95\% confidence interval (CI) to estimate the reliabilities of signatures were calculated by R package "pROC" ${ }^{12}$. To demonstrate the overview information of risk scores, patients' survival and expression of signature genes, data were listed in descending order by scaled risk scores and showed through R packages "ggplot2" and "heatmap" as previously described ${ }^{13}$. The results of univariable and multivariable Cox analysis of 7-RNA signature and clinical variables were visualized by the R package "forestplot".

\subsection{Nomogram and decision curve analysis (DCA)}


Three factors including our signature scores, age and tumor stage which were tightly associated with patients' survival in multivariable Cox analysis were selected, integrated and used to establish a new model predicting 3-year and 5-year survival of WT Bf-CM patients. Nomogram was drawn with the help of R package "rms". C-index and calibration curve were used to evaluate the predicting ability of nomogram. DCA is an effective method to estimate molecular signatures and prediction models, especially considering decision preference and clinical demands ${ }^{14}$. Visualization of DCA were achieved in the R statistics environment with the guide of "stdca" (https://github.com/mattblack/dcapy/blob/master/test/resource/stdca.R).

\subsection{Functional analysis}

To explore the function implications of the 7-RNA signature, the differently expression analysis between high risk groups and low risk groups were carried out using R package "edgeR" 15 . Differently expressed genes (DEG) were defined as absolute value of $\log _{2}$ FoldChange $>0.8$ and adjusted $p$ values $<0.001$. The potential interactions of 527 protein-coding DEGs were estimated in String and laid out using Cytoscape ${ }^{16,17}$. The application in Cytoscape Mcode was used to find the core gene module using criteria of Mcode scores ${ }^{18}$. The top biological process (BP) enrichment results of all DEGs and expression of involved genes were demonstrated and clustered by BP terms using $\mathrm{R}$ packages "GOplot" ${ }^{19}$. Gene set enrichment analysis (GSEA) on interested enrichment sets in MSigDB was based on R package "clusterProfiler" 20.

\subsection{Immune infiltration and drug prediction}

To investigate the immune microenvironment of WT Bf-CM tumor samples, 24 sorts of immune cells of each sample were quantified using single sample GSEA (SSGSEA) based on marker genes in previous study and immune infiltration was evaluated by the results of Hierarchical cluster ${ }^{21,22}$. Combination of immune infiltration results and clinical features was demodnstrated using $\mathrm{R}$ package "ComplexHeatmap" 23. As previously described, we used an R package "pRRophetic" to predict the inhibitory concentration 50 (IC50) of various anti-tumor drugs based on expression profiles ${ }^{24}$.

\section{Results}

\subsection{Clinical characteristics of the study populations}

A total of 211 WT Bf-CM patients' samples was downloaded from TCGA. As is shown in Table 1, $64.0 \%$ of the samples were collected from male and $36 \%$ were from female. The median age of this cohort is 62 , and the range of age is 25 87. 193 samples were matched with pathological stages according to the American Joint Committee on Cancer (AJCC) Cancer staging manual, the percentages of stage $0, \mathrm{I}, \mathrm{II}, \mathrm{III}$, IV were $1.9 \%, 16.6 \%, 29.9 \%, 39.3 \%$ and $3.8 \%$, respectively. $25.5 \%$ of these samples featured with a Breslow thickness $<2 \mathrm{~mm}, 28.5 \%$ within the range of $2 \mathrm{~mm}$ to $5 \mathrm{~mm}, 25.1 \%>5 \mathrm{~mm}$. More information of the selected clinical characteristics of are listed in Table 1. 


\subsection{Construction and validation of the expression-based prognostic signature}

In order to generate the training set and test set, $211 \mathrm{WT}$ Bf-CM patients were divided equally into two set at random, and their clinical characteristics are shown in Table 1. By analyzing the transcriptome data of training set with univariate Cox regression, 251 RNA were shown significantly to correspond with the 5year OS of WT Bf-CM patients ( $\mathrm{p} \otimes 0.01$ ). Next, multivariate Cox stepwise regression was performed based on the results of random forests and an expression-based prognostic signature was constructed. This prognostic signature is based on the hazard ratio of 7-RNA and can be formulated as: Risk scores = $0.31{ }^{*}$ expression of $\mathrm{CDC73}+0.282 *$ expression of RP1-69E11.3 + 0.882*expression of RP11-188D8.1+ $0.968^{*}$ expression of RP11-116P24.2+0.486*expression of TRIB2 $+0.203^{*}$ expression of VPS13D + 0.199*expression of CELF3. More information about the 7 RNA is listed in Table 2.

The risk scores of each patient from training set were calculated and the median score was used as cutoff for the stratification of high risk group and low risk group. Kaplan-Meier survival analysis and Logrank test of this two groups showed a significantly poorer prognostics of high risk group compared to low risk group (39.8 months vs. 170.2 months, $p<0.001$ ) (Figure.1a). For the prognostic value of the proposed signature, ROC curve of training set was drawn and AUC was calculated to be 0.866 (Figure.1a). To further validate the performance of the proposed signature, patients from test set were stratified similarly based on the median score from training set. Same analysis and tests were also performed with the highand low-risk groups of test set. Besides the significant correspondence between risk-score and prognosis (59.3 months vs. 90.4 months, $p=0.005)$, the ROC curve $(A U C=0.699)$ reveals the considerable potential of this signature for predicting the prognosis of WT Bf-CM patients in the test set (Figure.1b). Similar results were also observed in the entire WT Bf-CM set that high risk group had shorter OS than low risk group (47.9 months vs. 170.2 months, $p<0.001$ ) and the AUC was 0.780 (Figure.1c). Risk-related profiles revealed that more deaths occurred and expression of signature genes increased with the raise of risk scores (Figure.1d).

\subsection{Independence evaluation of the signature in prognostic prediction from clinical and pathological factors}

Traditional clinical and pathological factors have been well-clarified to be associated with the prognosis of melanoma patients, including age, gender, pathological stage, Breslow depths and radiation therapy 1,25. Using univariable and multivariable analysis, age and stage were shown to be significantly associated with the prognosis of $211 \mathrm{WT} \mathrm{Bf-CM}$ patients in addition to the proposed signature (Figure.2a). Therefore, age and stage were chosen to be compared with the proposed signature for its independence. As is shown in Figure.2b, when applied in each subgroup of entire set divided according to the risk stratification of each factor, the proposed signature revealed considerable performances in prognosis prediction. These results support the independence of this expression-based prognostic signature.

\subsection{Comparison of the signature with other known prognostic biomarkers}


Considering the burden brought by $\mathrm{CM}$, several studies have proposed other biomarkers for $\mathrm{CM}$ prognosis

prediction ${ }^{8,26-30}$. Although none of them specifically aimed at WT Bf-CM, we compared our signature with them to examine the predictive performances in WT Bf-CM prognosis. As is shown in Figure. 3a and Table. 3, the ROC analysis of time-dependent analysis in WT Bf-CM cohort proved that our signature had significant higher AUC than other biomarkers, and might be superior in WT Bf-CM prognosis prediction exclusively.

\subsection{Construction and evaluation of a prognostic nomogram for WT Bf-CM}

Nomogram has been a welcomed quantitative predictive tool for clinical practice. Based on the results from univariable and multivariable analysis above (Figure.2a), a nomogram to predict 3-year and 5-year survival of WT Bf-CM patients was constructed based on the risk scores of the proposed signature, age and pathological stage (Figure. 3b). The C-index of this nomogram was calculated to be 0.7505 and calibration plot was also drawn, exhibiting an acceptable accuracy for prediction (Figure. 3c). Additionally, DCA was performed to assess the efficiency of the proposed nomogram (Figure. 3d, e). When the threshold probability was lower than $25 \%$, our nomogram would be more beneficial and when threshold probability higher than $25 \%$, our signature would be more beneficial. These results supported the promising complementary use of our signature and nomogram.

\subsection{Functional analysis based on the signature grouping}

Cellular and molecular mechanism accounting for the prognosis of melanoma have always been a hot topic of cancer research. Thus, we aimed to find out what biological process might explain the predictive function of this signature. 865 genes were defined as DEGs between high risk and low risk group (Figure. 4a) and interactions of 527 proteins provided a landscape of potential molecular mechanisms (Figure. 4b). Using Mcode, a six-gene module with highest score (score 6) was selected as core module, which was closely associated with mRNA processing, splicing and metabolic process (Figure. 4b). BP enrichment analysis showed various processes were involved, including response to oxygen levels, RNA splicing, regulation of developmental growth, etc. (Figure. 4c). Besides, GSEA analysis indicated that the high-risk group was associated with the up-regulation of GO_NUCLEAR_SPECK (NES $=1.361, p=0.026)$, GO_PYRIMIDINE_NUCLEOTIDE_BIOSYNTHETIC_PROCESS (NES $=1.573, p=0.039)$,

REACTOME_MITOTIC_PROMETAPHASE (NES $=1.413, p=0.041)$ and TATA_01 (NES $=1.589, p=0.005)$ (Figure. 4d). Taken together, these results supported that the predictive function of this signature might be related to the up-regulation of RNA splicing, transcription, and cellular proliferation in the high-risk group, and upregulation of these processes have been demonstrated to be linked to malignancy of cancer ${ }^{31,32}$.

\subsection{Evaluation for the therapeutic responses based on signature grouping}

The treatment of cutaneous melanoma has made a considerable progress recently, especially for the application of immune-therapy and targeted drugs. Hence, we wondered if the proposed signature could provide clues for the therapeutic responses of WT Bf-CM patients. By estimating the immune infiltration of the WT Bf-CM cohort, we found that little differences in 24 sorts of immune cells infiltration (Figure. 
5a). Similarly, the comparison of the expression of 9 immune-markers showed little differences between high- and low- risk groups (Figure. 5b). Of note, although the checkpoint therapies targeting programmed cell death 1 (PD-1), programmed cell death-ligand 1 (PD-L1) and cytotoxic T lymphocyte antigen 4 (CTLA4) have been recommended for the treatment of WT Bf-CM, their expression didn't seem to be high both in high- or low-risk groups, suggesting the poor responses of checkpoint therapy in WT Bf-CM patients. Interestingly, the analysis of responses to chemo-therapies showed significant differences of 9 chemotherapeutic drugs in estimated IC50 between high and low-risk groups, along with an increased sensitivity to these 9 chemotherapies in high risk group (Figure. 5c). Among these 9 chemotherapeutic drugs, the targets of AKT.inhibitor.VIII, AS601245, GDC0941, MK.2206, PF.02341066 (Crizotinib),

Rapamycin and Temsirolimus are involved in the regulation of PI3K/Akt/mTOR pathway ${ }^{33-35}$, and this axis has known to play a critical role in cell proliferation and RNA processing 36,37 , further supporting the results from functional analysis. Altogether, these findings support that this signature could predict the responses of $\mathrm{PI3K} / \mathrm{Akt} / \mathrm{mTOR}$ pathway based drugs in WT Bf-CM patients, besides predicting their prognosis.

\section{Discussion}

As a highly heterogenous cancer, $\mathrm{CM}$ harbours various genetic alteration including BRAF mutation, RAS mutation, NF1 mutations, etc, which plays an critical role in the biological behavior of CM and is closely related to the prognosis of $\mathrm{CM}$ patient ${ }^{1}$. However, existing studies often discussed the prognosis or proposed predictive signatures of the total CM patients, rather than focus on CM patients with specific genetic alteration ${ }^{8,26-30}$, which might fail to give a precise result. Since the mutation status of BRAF has been used as a prognosis biomarker, and the prognosis of WT Bf-CM seems relatively poor owing to the lack of therapeutic options ${ }^{1-3,38}$, this study aimed to construct a predictive signature for the prognosis of WT Bf-CM patients. After extracting and the whole transcription profiles of tumor tissues from 211 WT BfCM patients in the TCGA database, we constructed a 7-RNA based signature in the training set. According to the $\mathrm{KM}$ analysis and ROC analysis, this signature exhibited a considerable performance in prognosis prediction both in the test set $(p=0.005$, AUC $=0.699)$ and entire set $(p<0.001, A U C=0.780)$.

In order to ensure the independency for prediction, signature should be compared with clinical factors contributing to the prognosis with stratified analysis. However, little is known about clinical factors that contributes to the prognosis of WT Bf-CM patients. Using univariate and multivariate Cox analysis among classic clinical factors related to CM prognosis ${ }^{25}$, we surprisedly found that only age and pathological stage were significantly correlated to the survival of WT Bf-CM patients. Although this might result from the limited size of our patients, it is of importance to make more exploration on the factors for WT Bf-CM prognosis. Subsequently, by stratifying the WT Bf-CM cohort according to age and pathological stage, the proposed signature was proven to be an independent predictive factor for the survival of WT Bf-CM prognosis. Next, by comparing the AUC curves, this signature exhibited a significant superior predictive performance exclusively for WT Bf-CM prognosis than other existing signatures for CM prognosis. In addition, to combine the predictive effect of this signature with clinical factors, a nomogram was drawn. 
Calibration and DCA analysis were performed and indicated a promising complementary use of our signature and nomogram. Given the poor understanding of factors associated with WT Bf-CM prognosis, the signature and nomogram in this study could make a more inspiring contribution to the precise management of WT Bf-CM.

Among the 7 RNA, CDC73 and TRIB2 have been identified as biomarkers for cancer prognosis 39,40 . Especially, TRIB2 could be used to indicate progression and the response to chemotherapy in ex vivo clinical samples of melanoma ${ }^{41}$. As for the other 2 mRNA, VPS13D and CELF3, some multi-omics or pathways based analysis indicated the their roles as potential drug target or biomarker for metastasis, but more experimental evidences are needed to further confirm their molecular roles in cancer biology 42,43 . Besides, little is known about the biological function of rest 3 ncRNA in cancer. Therefore, it is of significance to find some explanation of the biological mechanism for this signature. By comparing the transcription data between high- and low- risk groups, 865 DEGs were identified. Following analysis in protein interaction, biological process and pathway revealed that the up-regulation of mRNA splicing and processing, mitotic prometaphase, hypoxia-related metabolism alteration and pyrimidine nucleotide biosynthetic process were associated with the high-risk group. The former up-regulated processes are involved in the enhancement of cell proliferation and are linked to malignancy of cancer ${ }^{32}$, which means the poor survival of WT Bf-CM predicted by this signature might be resulted from the more malignant behavior in cancer biology. This was supported by a retrospective study which concluded that low tumor proliferation rate was significantly associated with a better prognosis ${ }^{44}$. Moreover, in spite of the removal from sub-classification of thin melanomas in recent the AJCC staging system ${ }^{45}$, mitotic rate can still be a significant prognostic indicator especially for WT Bf-CM.

As a cancer with high mortality, the survival of CM patients largely depends on the response to therapies. To date, immune checkpoint blockage therapy has been recommended for the treatment of advanced WT $\mathrm{Bf}-\mathrm{CM}^{46}$. Additionally, the infiltration of immune cells can both serve as the predictor of responses to checkpoint therapy and WT Bf-CM survival $44,47,48$. However, little differences in immune infiltration was observed in the WT Bf-CM patients between high- and low- risk groups. Also, the expression of immune checkpoint such as PD-1, PD-L1 and CTLA-4, was relatively low expressed in WT Bf-CM patients, indicating the insensitivity of WT Bf-CM towards checkpoint therapy. Thus, we performed the analysis of responses to chemo-therapies and identified 9 drugs that might be effective for WT Bf-CM. Most of the 9 drugs function by targeting PI3K/Akt/mTOR axis. The axis of PI3K/Akt/mTOR, however, has been wellclarified as a key regulator in RNA processing, cellular proliferation and metabolic reprogramming, corresponding to the previous results in functional analysis in DEGs ${ }^{36,37,49} .3$ of the RNA for signature construction, including CDC73, TRIB2, VPS13D are, also involved in the regulatory network of $\mathrm{PI} 3 \mathrm{~K} / \mathrm{Akt} / \mathrm{mTOR}$, which have been confirmed in functional experiments ${ }^{42,50,51}$. Inhibiting PI3K/Akt/mTOR could even have a therapeutic effect for $\mathrm{CM}$ when resistance to BRAF inhibition formed, suggesting that targeting PI3K/Akt/mTOR could treat CM independent of BRAF status ${ }^{52}$. Considering that some of the drugs targeting PI3K/Akt/mTOR, including PF.02341066, Rapamycin and Temsirolimus, have been 
approved for clinical practice in cancer ${ }^{53,54}$, the perspective for their use in improving WT Bf-CM prognosis is bright.

Nevertheless, there are deficiencies with the present study. First, since it is difficult to find a cohort contain the corresponsive data of BRAF status, transcription and survival, this signature was not validated with external cohort. Second, simple cross validation might cause the problem of overfitting and weaken the ability of generalization. However, random forest is fundamentally based on independent model ensemble and adept in generalizing and avoiding overfitting. Insufficient trees were downvoted and testing set actually exerted a positive effect on selecting efficient results to eliminate out of bagging. Third, no experimental evidence supports the potential mechanisms of the present signature and further experiments might reveal the underlying molecular interactions.

\section{Conclusion}

Collectively, this study proposed a novel 7-RNA signature for the prognostic prediction of WT Bf-CM. As far as we are concerned, this is the first prognostic signature for WT Bf-CM, which might provide more assistance in the management of WT Bf-CM patients.

\section{Abbreviations}

WT Bf-CM, wild-type BRAF cutaneous melanoma

$\mathrm{CM}$, cutaneous melanoma

PFS, progression-free survival

NGS, next-generation sequencing

RPKM, Reads Per Kilobase per Million mapped reads

OS, overall survival

ROC, receiver operating characteristic

AUC, the area under ROC

DCA, decision curve analysis

DEG, differently expressed genes

GESA, gene set enrichment analysis

BP, biological process

IC50, inhibitory concentration 50 
PD-1, programmed cell death 1 ,

PD-L1, programmed cell death-ligand 1

CTLA-4, cytotoxic T lymphocyte antigen 4

\section{Declarations}

\section{Funding}

This research did not receive any specific grant from funding agencies in the public, commercial, or notfor-profit sectors.

\section{Acknowledgement}

We particularly thank the support and care in academic spirits from Di Fu (Shanghai Jiao Tong University School of Medicine) and Qi Qin (Sun Yat-sen University Zhongshan School of Medicine).

\section{Competing Interest}

The authors declare no conflicts of interest.

\section{Ethics approval}

No ethics approval should be declared.

\section{Consent to participate}

No applicable.

\section{Consent for publication}

All the involved members agreed for publication.

\section{Availability of data and material}

All the data can be downloaded from https://portal.gdc.cancer.gov/.

\section{Code availability}

All the code can be guided by manuscripts of corresponding R packages.

\section{Author Contributions Statement}

Data collection: R.S.,L.L.; study design: Z. T.,L.L.; writing: Z. T., C.L and R. S; data analysis: R.S., Y. L and Z.T.L.L.; data interpretation/revision/final approval: all authors. 


\section{References}

1. Schadendorf D, van Akkooi ACJ, Berking C, et al. Melanoma. The Lancet. 2018;392(10151):971-984.

2. Verduzco D, Kuenzi BM, Kinose F, et al. Ceritinib Enhances the Efficacy of Trametinib in BRAF/NRASWild-Type Melanoma Cell Lines. Mol Cancer Ther. 2018;17(1):73-83.

3. Capparelli C, Purwin TJ, Heilman SA, et al. ErbB3 Targeting Enhances the Effects of MEK Inhibitor in Wild-Type BRAF/NRAS Melanoma. Cancer Res. 2018;78(19):5680-5693.

4. Frankel AE, Eskiocak U, Gill JG, et al. Digoxin Plus Trametinib Therapy Achieves Disease Control in BRAF Wild-Type Metastatic Melanoma Patients. Neoplasia. 2017;19(4):255-260.

5. Falchook GS, Lewis KD, Infante JR, et al. Activity of the oral MEK inhibitor trametinib in patients with advanced melanoma: a phase 1 dose-escalation trial. Lancet Oncol. 2012;13(8):782-789.

6. Dummer R, Schadendorf D, Ascierto PA, et al. Binimetinib versus dacarbazine in patients with advanced NRAS-mutant melanoma (NEMO): a multicentre, open-label, randomised, phase 3 trial. Lancet Oncol. 2017;18(4):435-445.

7. Goodman AM, Kato S, Bazhenova L, et al. Tumor Mutational Burden as an Independent Predictor of Response to Immunotherapy in Diverse Cancers. Mol Cancer Ther. 2017;16(11):2598-2608.

8. Guo W, Zhu L, Zhu R, Chen Q, Wang Q, Chen JQ. A four-DNA methylation biomarker is a superior predictor of survival of patients with cutaneous melanoma. Elife. 2019;8.

9. Jiang $Y$, Shi $X$, Zhao Q, Krauthammer M, Rothberg BE, Ma S. Integrated analysis of multidimensional omics data on cutaneous melanoma prognosis. Genomics. 2016;107(6):223-230.

10. Yang $\mathrm{S}, \mathrm{Xu} \mathrm{J}$, Zeng X. A six-long non-coding RNA signature predicts prognosis in melanoma patients. Int J Oncol. 2018;52(4):1178-1188.

11. Colaprico A, Silva TC, Olsen C, et al. TCGAbiolinks: an R/Bioconductor package for integrative analysis of TCGA data. Nucleic Acids Res. 2016;44(8):e71.

12. Robin X, Turck N, Hainard A, et al. pROC: an open-source package for R and S+ to analyze and compare ROC curves. BMC Bioinformatics. 2011;12:77.

13. Li X, Shi Y, Yin Z, Xue X, Zhou B. An eight-miRNA signature as a potential biomarker for predicting survival in lung adenocarcinoma. $J$ Trans/ Med. 2014;12:159.

14. Vickers AJ, Cronin AM, Elkin EB, Gonen M. Extensions to decision curve analysis, a novel method for evaluating diagnostic tests, prediction models and molecular markers. BMC Med Inform Decis Mak. 2008;8:53.

15. Robinson MD, McCarthy DJ, Smyth GK. edgeR: a Bioconductor package for differential expression analysis of digital gene expression data. Bioinformatics. 2010;26(1):139-140.

16. Szklarczyk D, Gable AL, Lyon D, et al. STRING v11: protein-protein association networks with increased coverage, supporting functional discovery in genome-wide experimental datasets. Nucleic Acids Res. 2019;47(D1):D607-D613. 
17. Shannon P, Markiel A, Ozier O, et al. Cytoscape: a software environment for integrated models of biomolecular interaction networks. Genome Res. 2003;13(11):2498-2504.

18. Bader GD, Hogue CW. An automated method for finding molecular complexes in large protein interaction networks. BMC Bioinformatics. 2003;4:2.

19. Walter W, Sanchez-Cabo F, Ricote M. GOplot: an R package for visually combining expression data with functional analysis. Bioinformatics. 2015;31(17):2912-2914.

20. Yu G, Wang LG, Han Y, He QY. clusterProfiler: an R package for comparing biological themes among gene clusters. OMICS. 2012;16(5):284-287.

21. Bindea G, Mlecnik B, Tosolini M, et al. Spatiotemporal dynamics of intratumoral immune cells reveal the immune landscape in human cancer. Immunity. 2013;39(4):782-795.

22. Hanzelmann S, Castelo R, Guinney J. GSVA: gene set variation analysis for microarray and RNA-seq data. BMC Bioinformatics. 2013;14:7.

23. Gu Z, Eils R, Schlesner M. Complex heatmaps reveal patterns and correlations in multidimensional genomic data. Bioinformatics. 2016;32(18):2847-2849.

24. Geeleher P, Cox N, Huang RS. pRRophetic: an R package for prediction of clinical chemotherapeutic response from tumor gene expression levels. PLoS One. 2014;9(9):e107468.

25. Garbe C, Amaral T, Peris K, et al. European consensus-based interdisciplinary guideline for melanoma. Part 1: Diagnostics - Update 2019. European Journal of Cancer. 2020;126:141-158.

26. Chen X, Guo W, Xu XJ, et al. Melanoma long non-coding RNA signature predicts prognostic survival and directs clinical risk-specific treatments. J Dermato/ Sci. 2017;85(3):226-234.

27. Shi G, Li H, Gao F, Tan Q. IncRNA H19 predicts poor prognosis in patients with melanoma and regulates cell growth, invasion, migration and epithelial-mesenchymal transition in melanoma cells. Onco Targets Ther. 2018;11:3583-3595.

28. Timani KA, Gyorffy B, Liu Y, Mohammad KS, He JJ. Tip110/SART3 regulates IL-8 expression and predicts the clinical outcomes in melanoma. Mol Cancer. 2018;17(1):124.

29. Wang Q, Wang X, Liang Q, et al. Prognostic Value of Dynactin mRNA Expression in Cutaneous Melanoma. Med Sci Monit. 2018;24:3752-3763.

30. Zhang W, Li X, Song G, Luo D. Prognostic significance of LKB1 promoter methylation in cutaneous malignant melanoma. Oncol Lett. 2017;14(2):2075-2080.

31. Dong X, Chen R. Understanding aberrant RNA splicing to facilitate cancer diagnosis and therapy. Oncogene. 2020;39(11):2231-2242.

32. Loberg RD, Bradley DA, Tomlins SA, Chinnaiyan M, Pieta KJ. The lethal phenotype of cancer: The molecular basis of death due to malignancy. Ca-Cancer J Clin. 2007;57(4):225-241.

33. Hu N, Zhang Y. TLR4 knockout attenuated high fat diet-induced cardiac dysfunction via NFkappaB/JNK-dependent activation of autophagy. Biochim Biophys Acta Mol Basis Dis. 2017;1863(8):2001-2011. 
34. Li J, Xiao Q, Bao Y, et al. HER2-L755S mutation induces hyperactive MAPK and PI3K-mTOR signaling, leading to resistance to HER2 tyrosine kinase inhibitor treatment. Cell Cycle. 2019;18(13):1513-1522.

35. Ji S, Lin W, Wang L, et al. Combined Targeting of mTOR and Akt Using Rapamycin and MK-2206 in The Treatment of Tuberous Sclerosis Complex. J Cancer. 2017;8(4):555-562.

36. Davis WJ, Lehmann PZ, Li W. Nuclear PI3K signaling in cell growth and tumorigenesis. Front Cell Dev Biol. 2015;3:24.

37. White RJ, Sharrocks AD. Coordinated control of the gene expression machinery. Trends Genet. 2010;26(5):214-220.

38. Ny L, Hernberg M, Nyakas M, et al. BRAF mutational status as a prognostic marker for survival in malignant melanoma: a systematic review and meta-analysis. Acta Oncol. 2020:1-12.

39. Zheng HC, Gong BC, Zhao S. The clinicopathological and prognostic significances of CDC73 expression in cancers: a bioinformatics analysis. Oncotarget. 2017;8(56):95270-95279.

40. Wang J, Zuo J, Wahafu A, Wang MD, Li RC, Xie WF. Combined elevation of TRIB2 and MAP3K1 indicates poor prognosis and chemoresistance to temozolomide in glioblastoma. CNS Neurosci Ther. 2020;26(3):297-308.

41. Hill R, Kalathur RK, Colaco L, et al. TRIB2 as a biomarker for diagnosis and progression of melanoma. Carcinogenesis. 2015;36(4):469-477.

42. Andersen JN, Sathyanarayanan S, Di Bacco A, et al. Pathway-based identification of biomarkers for targeted therapeutics: personalized oncology with PI3K pathway inhibitors. Sci Trans/ Med. 2010;2(43):43ra55.

43. Zhou B, Guo R. Integrative analysis of significant RNA-binding proteins in colorectal cancer metastasis. J Cell Biochem. 2018;119(12):9730-9741.

44. Falkenius J, Johansson H, Tuominen R, Frostvik Stolt M, Hansson J, Egyhazi Brage S. Presence of immune cells, low tumor proliferation and wild type BRAF mutation status is associated with a favourable clinical outcome in stage III cutaneous melanoma. BMC Cancer. 2017;17(1):584.

45. Gershenwald JE, Scolyer RA, Hess KR, et al. Melanoma staging: Evidence-based changes in the American Joint Committee on Cancer eighth edition cancer staging manual. CA Cancer $J$ Clin. 2017;67(6):472-492.

46. Kohn CG, Zeichner SB, Chen Q, Montero AJ, Goldstein DA, Flowers CR. Cost-Effectiveness of Immune Checkpoint Inhibition in BRAF Wild-Type Advanced Melanoma. J Clin Oncol. 2017;35(11):1194-1202.

47. Jessurun CAC, Vos JAM, Limpens J, Luiten RM. Biomarkers for Response of Melanoma Patients to Immune Checkpoint Inhibitors: A Systematic Review. Front Oncol. 2017;7:233.

48. Kumpers C, Jokic M, Haase O, et al. Immune Cell Infiltration of the Primary Tumor, Not PD-L1 Status, Is Associated With Improved Response to Checkpoint Inhibition in Metastatic Melanoma. Front Med (Lausanne). 2019;6:27.

49. Saxton RA, Sabatini DM. mTOR Signaling in Growth, Metabolism, and Disease. Cell. 2017;168(6):960-976. 
50. Zhang JH, Seigneur EM, Pandey M, et al. The EIF4EBP3 translational repressor is a marker of CDC73 tumor suppressor haploinsufficiency in a parathyroid cancer syndrome. Cell Death Dis. 2012;3:266.

51. Hill R, Madureira PA, Ferreira B, et al. TRIB2 confers resistance to anti-cancer therapy by activating the serine/threonine protein kinase AKT. Nat Commun. 2017;8:14687.

52. Deng W, Gopal YN, Scott A, Chen G, Woodman SE, Davies MA. Role and therapeutic potential of PI3KmTOR signaling in de novo resistance to BRAF inhibition. Pigment Cell Melanoma Res. 2012;25(2):248-258.

53. Benjamin D, Colombi M, Moroni C, Hall MN. Rapamycin passes the torch: a new generation of mTOR inhibitors. Nat Rev Drug Discov. 2011;10(11):868-880.

54. Roys A, Chang X, Liu Y, Xu X, Wu Y, Zuo D. Resistance mechanisms and potent-targeted therapies of ROS1-positive lung cancer. Cancer Chemother Pharmacol. 2019;84(4):679-688.

\section{Tables}

\section{Table 1. Clinico-pathological characteristics of WT Bf-CM patients}




\begin{tabular}{|c|c|c|c|}
\hline Groups & $\begin{array}{l}\text { Total } \\
(\mathrm{N}=211)\end{array}$ & $\begin{array}{l}\text { Training } \\
\text { Group }(\mathrm{N}=106)\end{array}$ & $\begin{array}{l}\text { Test Group } \\
(\mathrm{N}=105)\end{array}$ \\
\hline \multicolumn{4}{|l|}{ Gender } \\
\hline Male & $135(64.0 \%)$ & 73(68.9\%) & $62(59.0 \%)$ \\
\hline Female & 76(36.0\%) & $33(31.1 \%)$ & $43(41.0 \%$ \\
\hline \multicolumn{4}{|l|}{ Age At Diagnosis } \\
\hline Median & 62 & 63.5 & 61 \\
\hline Range & $25 \sim 87$ & $30 \sim 87$ & $25 \sim 83$ \\
\hline$\leq 58$ & $90(42.7 \%)$ & $42(39.6 \%)$ & $48(45.7 \%)$ \\
\hline$\bigotimes 58$ & $121(57.3 \%)$ & $64(60.3 \%)$ & $57(54.3 \%)$ \\
\hline \multicolumn{4}{|l|}{ Tumor Tissue Site } \\
\hline Primary Tumor & $38(18.0 \%)$ & $20(18.9 \%)$ & $18(17.10 \%)$ \\
\hline $\begin{array}{l}\text { Regional Cutaneous Or Subcutaneous } \\
\text { Tissue }\end{array}$ & $38(18.0 \%)$ & $21(19.8 \%)$ & $17(16.20 \%)$ \\
\hline Regional Lymph Node Metastasis & $93(44.1 \%)$ & $46(43.4 \%)$ & $47(44.80 \%)$ \\
\hline Distant Metastasis & $40(19.0 \%)$ & $18(17.0 \%)$ & $22(21.00 \%)$ \\
\hline Unknown & $2(0.9 \%)$ & $1(0.9 \%)$ & $1(1.00 \%)$ \\
\hline \multicolumn{4}{|l|}{ Pathological Stage } \\
\hline 0 & $4(1.9 \%)$ & $1(0.9 \%)$ & $3(2.90 \%)$ \\
\hline I & $35(16.6 \%)$ & $13(12.3 \%)$ & $22(21.00 \%)$ \\
\hline ॥ & $63(29.9 \%)$ & $33(31.1 \%)$ & $30(28.60 \%)$ \\
\hline III & $83(39.3 \%)$ & $43(40.6 \%)$ & $40(38.10 \%)$ \\
\hline IV & $8(3.8 \%)$ & $4(3.8 \%)$ & $4(3.80 \%)$ \\
\hline Unknown & $18(8.5 \%)$ & $12(11.3 \%)$ & $6(5.70 \%)$ \\
\hline \multicolumn{4}{|l|}{ Anatomic Site } \\
\hline Head And Neck & $16(7.6 \%)$ & $8(7.5 \%)$ & $8(7.60 \%)$ \\
\hline Extremities & 108(51.2\%) & $55(51.9 \%)$ & $53(50.50 \%)$ \\
\hline Trunk & $56(26.5 \%)$ & $28(26.4 \%)$ & $28(26.70 \%)$ \\
\hline Others/Unknown & $31(14.7 \%)$ & $15(14.2 \%)$ & $16(15.20 \%)$ \\
\hline
\end{tabular}




\begin{tabular}{|llll|}
\hline 2 & $54(25.5 \%)$ & $26(24.6 \%)$ & $28(26.70 \%)$ \\
\hline $2 \sim 5$ & $60(28.5 \%)$ & $36(33.9 \%)$ & $24(22.80 \%)$ \\
\hline 5 & $53(25.1 \%)$ & $25(23.6 \%)$ & $28(26.70 \%)$ \\
\hline Unknown & $44(20.9 \%)$ & $19(17.9 \%)$ & $25(23.80 \%)$ \\
\hline Radiation Therapy & & & $17(16.20 \%)$ \\
\hline Yes & $37(17.5 \%)$ & $20(18.9 \%)$ & $80(76.20 \%)$ \\
\hline No & $155(73.5 \%)$ & $75(70.8 \%)$ & $8(7.60 \%)$ \\
\hline Unknown & $19(9.00 \%)$ & $11(10.40 \%)$ & $45(42.90 \%)$ \\
\hline Vital Status & & & $60(57.10 \%)$ \\
\hline Yes & $81(38.4 \%)$ & $36(34.0 \%)$ & $70(66.0 \%)$ \\
\hline No & $130(61.6 \%)$ & & \\
\hline
\end{tabular}

Table 2. 7 RNAs significantly associated with the overall survival

\begin{tabular}{llllll} 
Gene symbol & Gene ID & Gene_type & Coeffcient & HR & pvalue \\
\hline CDC73 & ENSG00000134371 & protein coding & 0.318 & 1.374 & 0.015 \\
\hline RP1-69E11.3 & ENSG00000237131 & processed pseudogene & 0.282 & 1.326 & 0.044 \\
\hline RP11-188D8.1 & ENSG00000271427 & lincRNA & 0.882 & 2.416 & 0.013 \\
\hline RP11-116P24.2 & ENSG00000281535 & lincRNA & 0.968 & 2.633 & 0.040 \\
\hline TRIB2 & ENSG00000071575 & protein coding & 0.486 & 1.625 & 0.012 \\
\hline VPS13D & ENSG00000048707 & protein coding & 0.203 & 1.225 & 0.034 \\
\hline CELF3 & ENSG00000159409 & protein coding & 0.199 & 1.220 & 0.108
\end{tabular}

Table 3. The ROC results of our signature and other latest biomarkers of SKCM 


\begin{tabular}{llllll} 
Signature & AUC & $\mathbf{9 5 \%}$ Cl of AUC & Type & P-value $^{1}$ & Reference \\
\hline Our signature & 0.780 & $0.70-0.87$ & LncRNA and mRNA & & This study \\
\hline Four-IncRNA & 0.536 & $0.43-0.64$ & LncRNA & 0.000 & 26 \\
\hline H19 & 0.550 & $0.44-0.66$ & LncRNA & 0.000 & 27 \\
\hline SART3 & 0.572 & $0.47-0.68$ & Protein coding & 0.002 & 28 \\
\hline DCTN1 & 0.528 & $0.46-0.60$ & Protein coding & 0.000 & 29 \\
\hline STK11 & 0.560 & $0.45-0.67$ & Methylation & 0.001 & 30 \\
\hline Four-DNA methylation & 0.550 & $0.44-0.66$ & Methylation & 0.002 & 8
\end{tabular}

1 -value of AUC value comparisons between our signature and other latest biomarkers.

\section{Figures}


a

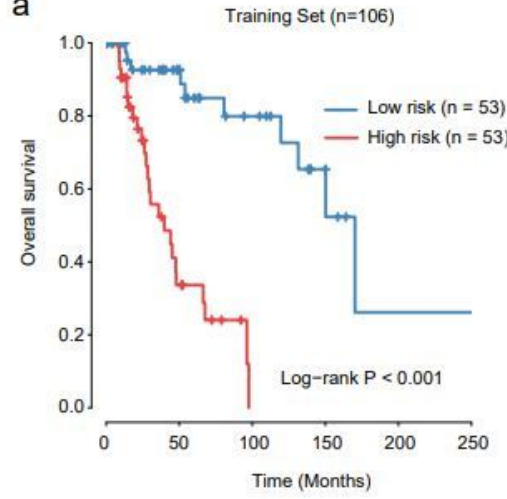

b
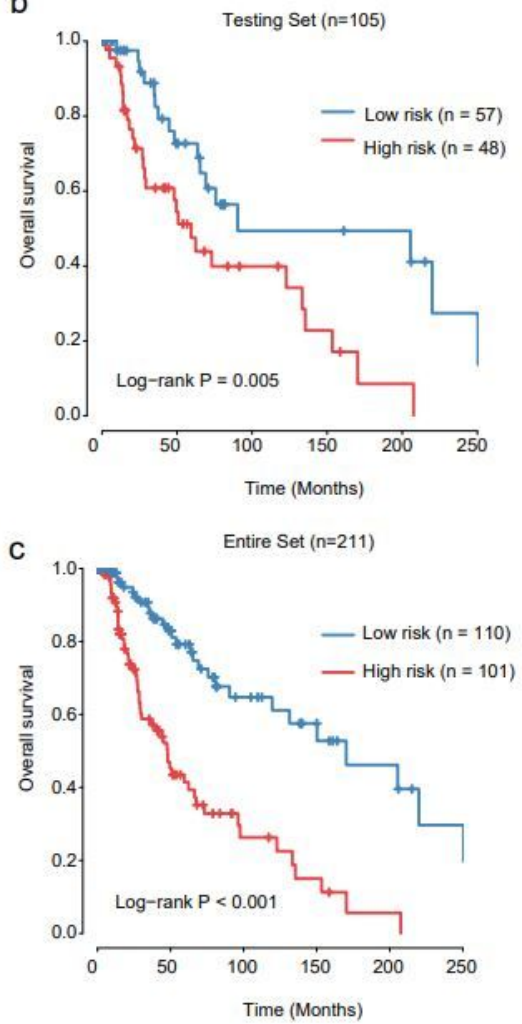

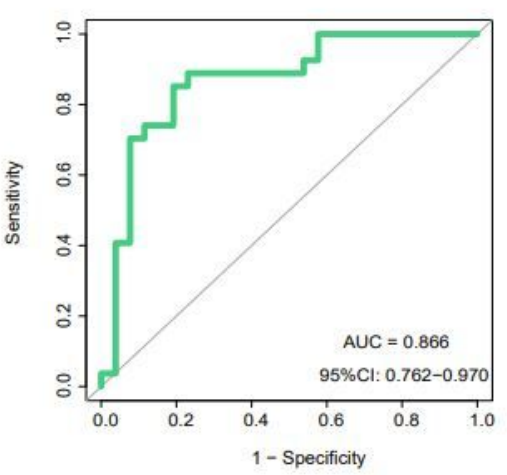

d
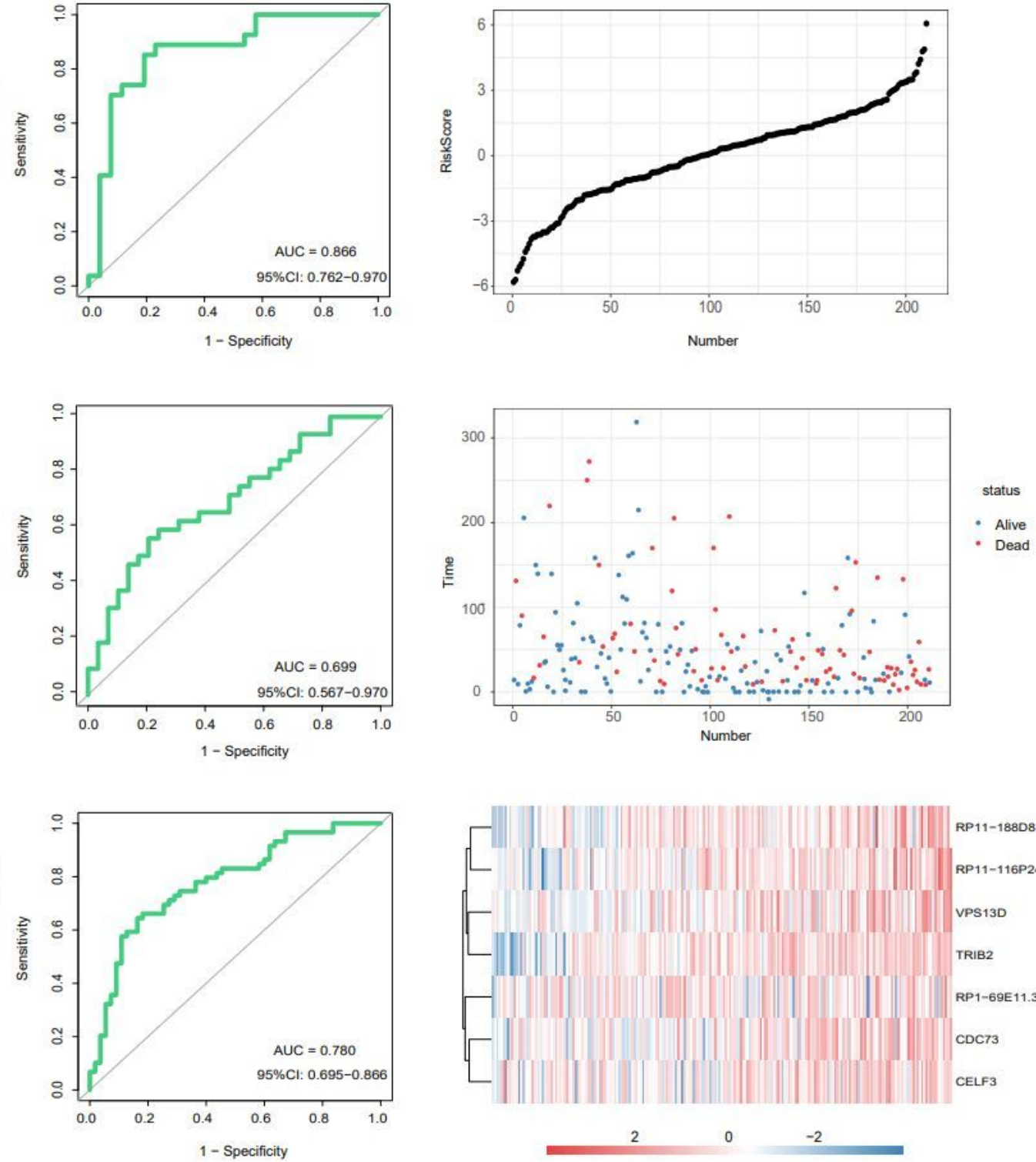

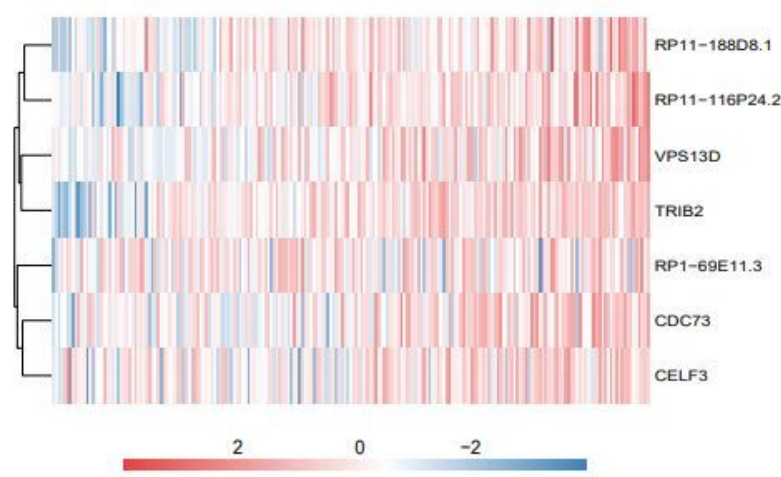

\section{Figure 1}

The 7-RNA signature-related risk score and OS prediction of WT Bf-CM patients. KM analysis reveal the OS differences between high and low risk groups and ROC curves assess the predictive performance of the signature-related score in training set (a); in testing set (b) and in the entire set (c). P values were calculated by two-sided log-rank tests, total AUC values were estimated and $95 \% \mathrm{Cl}$ were computed with 2000 stratified bootstrap replicates. (d) Risk-related plots illustrate the risk scores, survival status of patients and heatmap of 7 RNAs' expression. 


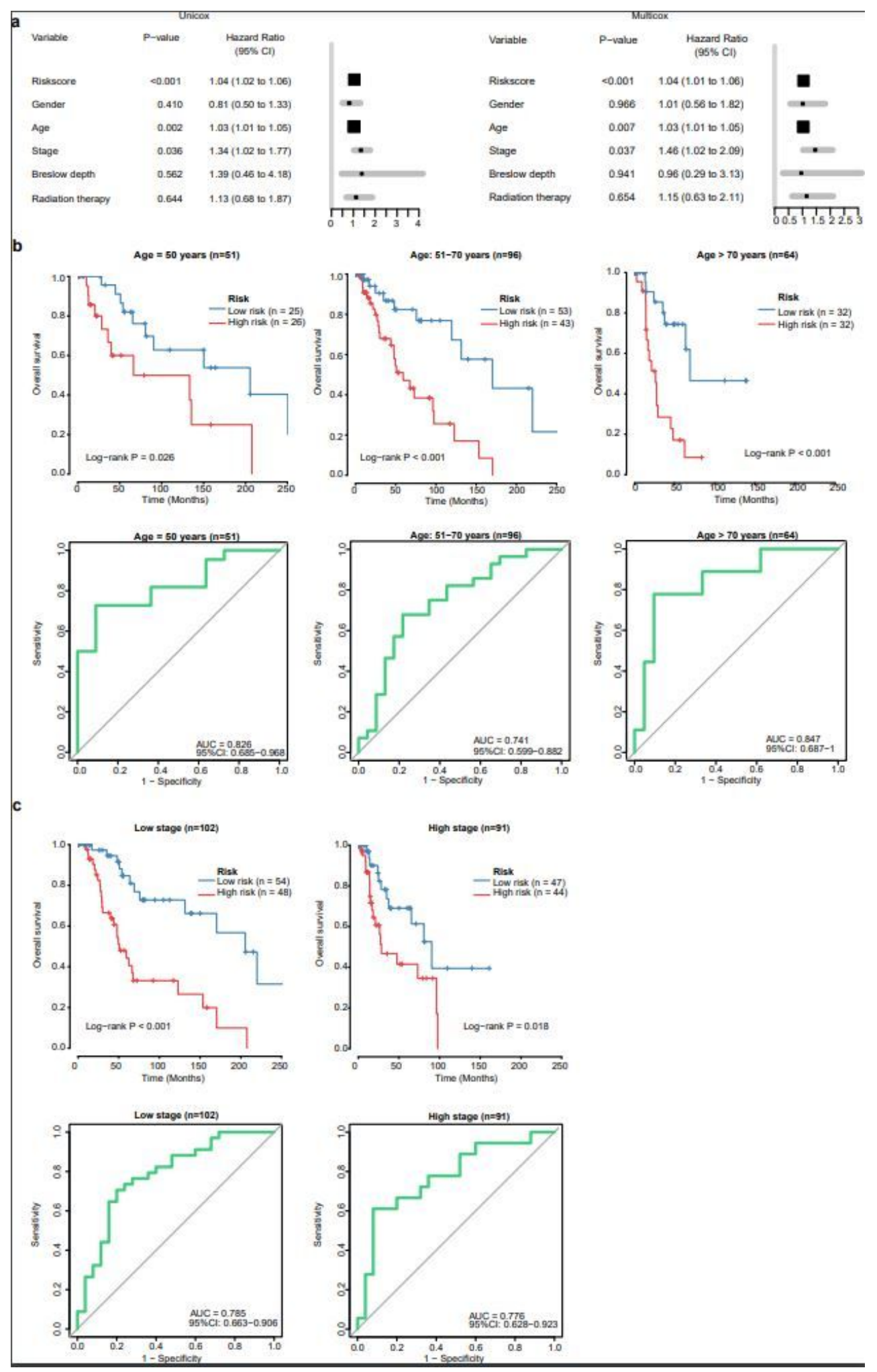

Figure 2

Signature's independence of clinical factors and stratification analysis. Univariable Cox regression and multivariable Cox regression analysis were performed, P-value (significance), Hazard Ratio and $95 \% \mathrm{Cl}$ were respectively shown in (a) and (b). KM and ROC analysis of regrouping cohorts based on age and stage were correspondingly demonstrated in (c) and (d). 
a

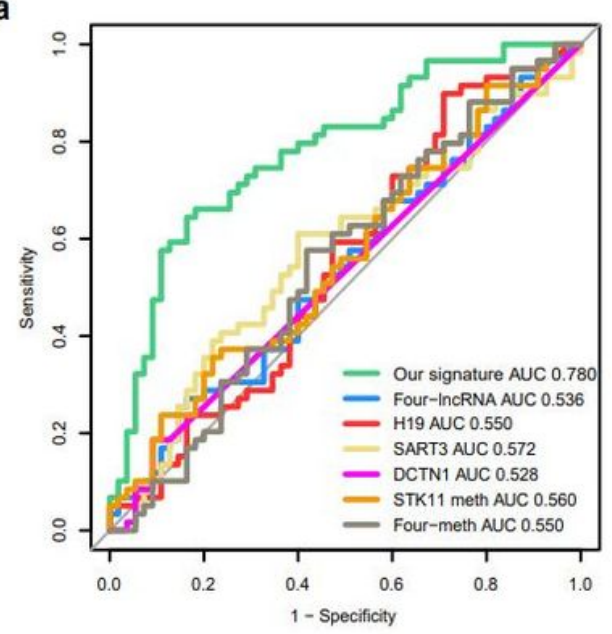

b

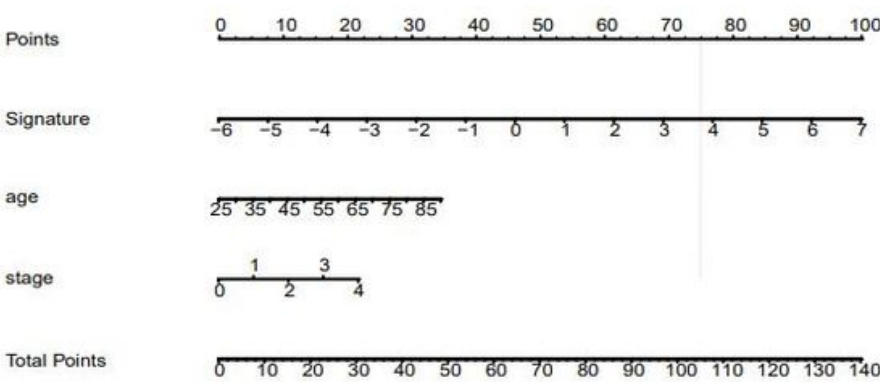

3-year survival Probability

5-year survival Probability

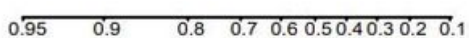

$0.95 \quad 0.9 \quad 0.8 \quad 0.7 \quad 0.60 .50 .40 .30 .20 .1$
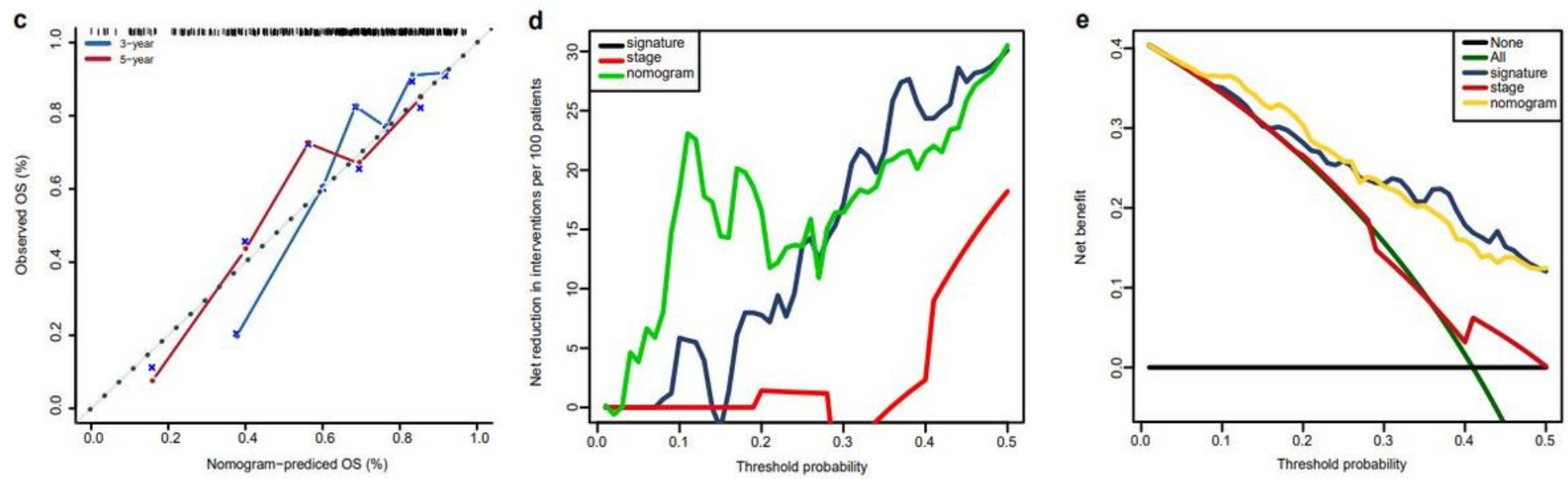

Figure 3

(a) Comparison of predictive performances of our 7-RNA signature and other known biomarkers/signatures. (b) The signature-based nomogram to predict 3-year and 5-year survival probability. (c) The calibration plot of nomogram predicted and real surviving proportions. (d) Net reduction interventions and $(E)$ net benefit of decision curve analysis. 
a

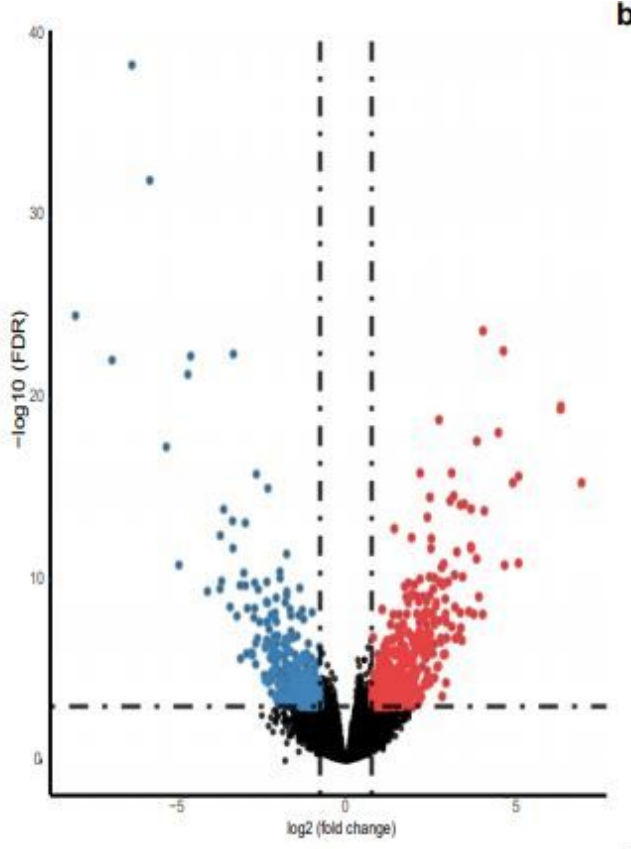

c

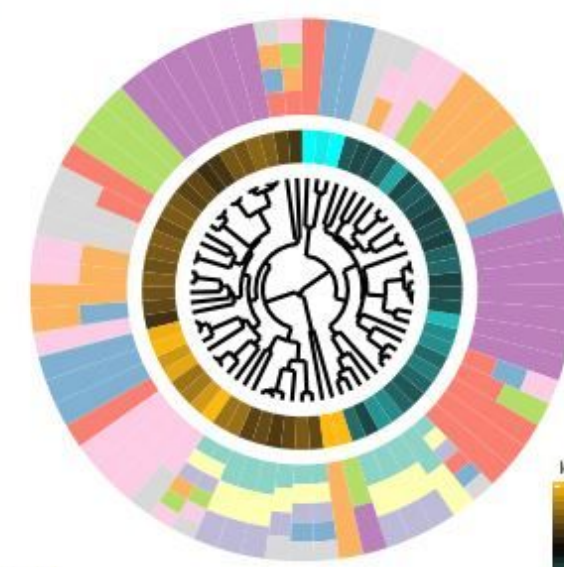

GO Terms

$\square$ response to decreased axygen levels $\square$ regulation of developmental growth $\square$ regulation of epithelial cell proliferation $\square$ response to oxygen levels $\square$ regulation of intracellular transport $\square$ RNA splicing b

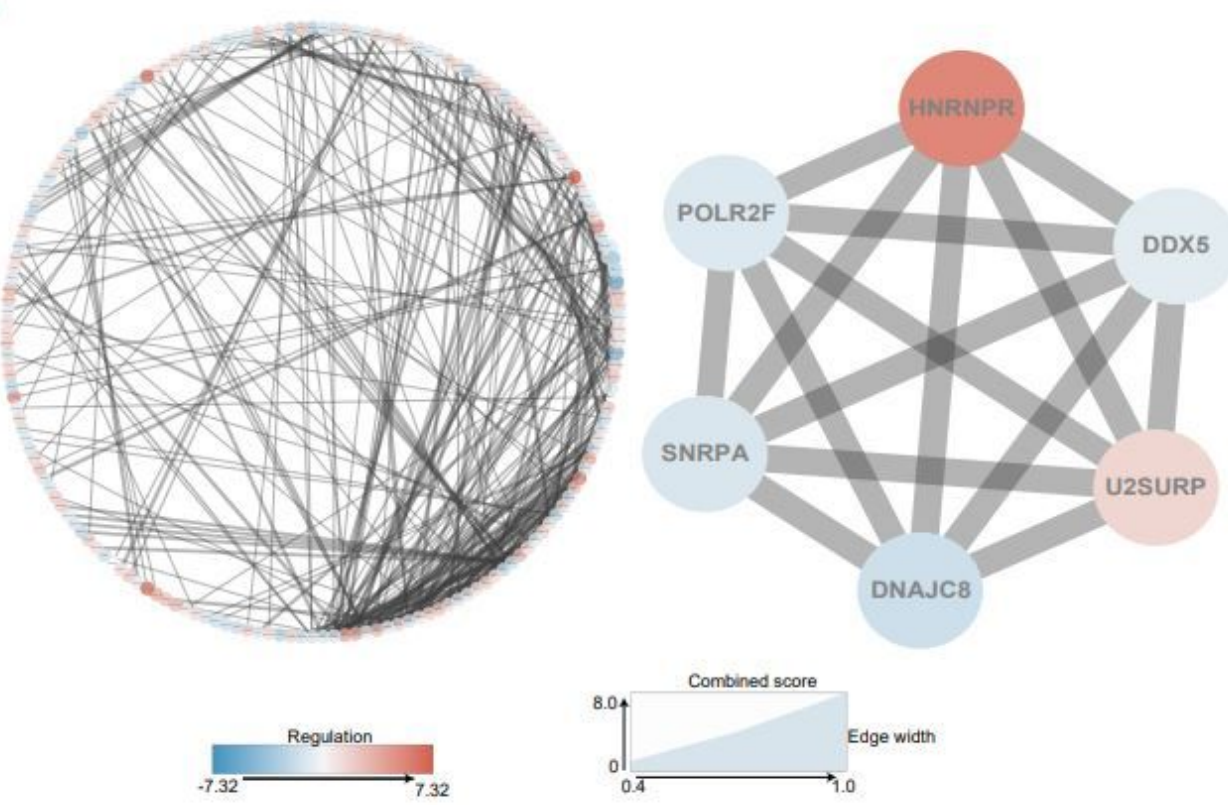

d
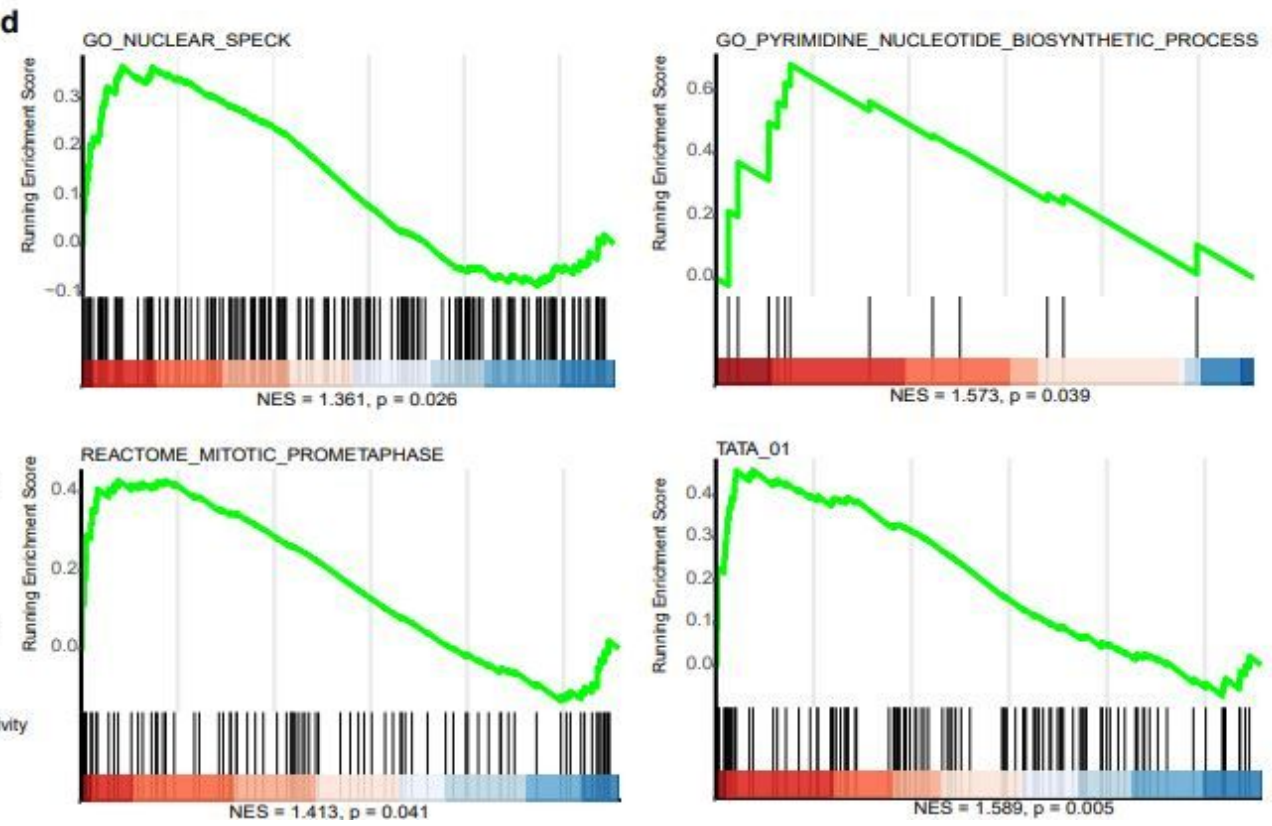

Figure 4

(a) Volcano plot of DEGs between high risk and low risk group. (b) Protein-protein interactions (PPI) of DEGs in String and core module of PPI with the highest Mcode score. (c) GOplot of BP enrichment results showing term-clustered gene set and fold changes of genes. (d) GSEA results of specific enrichment set in MSigDB. 


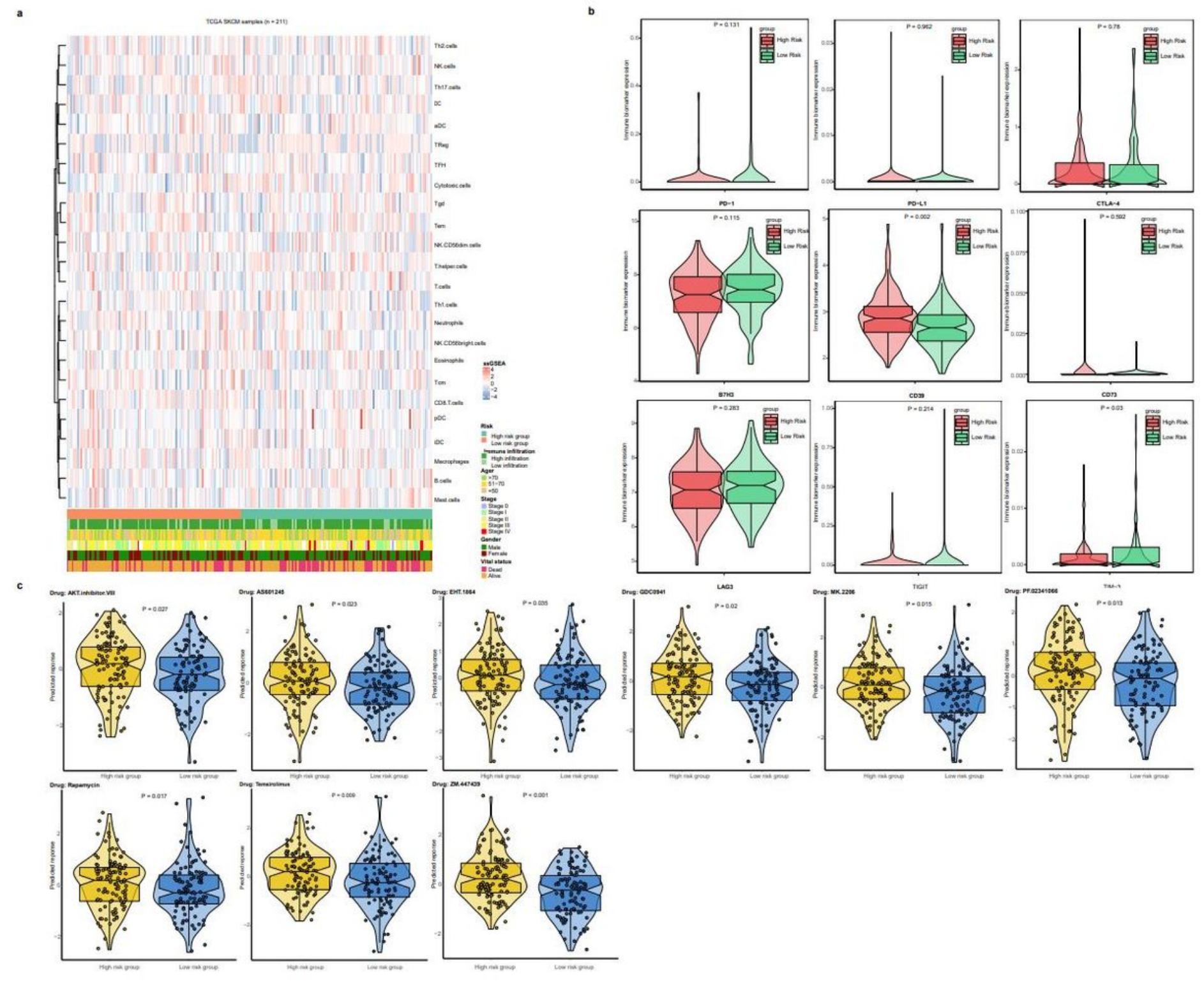

Figure 5

(a) Complex heatmap of 24 sorts of immune cells of WT Bf-CM patients, accompanied by signaturerelated risk groups, immune infiltration, age, stage, gender and vital status. (b) Expression of immune markers in high and low risk groups. (c) Predicted IC50 of various anti-cancer drugs. The $p$ values were calculated using Wilcoxon test. 